\title{
Redes de gestión compartida entre organizaciones de la sociedad civil en la localidad de Ciudad Bolívar, 2005-2006. Un insumo para la política pública en Bogotá D.C.
}

\author{
Leonardo Garavito González - Universidad Externado de Colombia ${ }^{1}$
}

\begin{abstract}
Resumen
El siguiente artículo presenta los resultados básicos de un análisis de redes sociales - ARS- aplicado entre organizaciones de la sociedad civil que trabajan en la localidad de Ciudad Bolívar, en la ciudad de Bogotá D.C. En este ejercicio se observaron sus vínculos entorno a la gestión de acciones y proyectos compartidos para el periodo 2005-2006. Este análisis pretende ser un insumo para la formulación y aplicación de una política pública distrital que busca la creación de una Red Distrital de Reconciliación para la ciudad. A través del ejercicio se encontró que en Ciudad Bolívar existe una red de organizaciones relativamente pequeña pero potencial para los objetivos de dicha política pública.
\end{abstract}

Palabras clave: Redes Sociales - Organizaciones de la Sociedad Civil - Ciudad Bolívar - Bogotá D.C.

\section{Abstract}

The article presents a social network analysis that was applied to civil organizations working in Ciudad Bolivar, which is a locality in Bogotá D.C. The exercise is focused on the working ties of sharing actions and projects during the years 2005 and 2006. The research aims to be an input for the local public policy of Bogotá D.C., which wants to create the reconciliation district network. The main results demonstrate the existence of a small but potential social network that could be a starting point to the achievement of public policy goals.

Key words: Social Networks - Civil Organizations - Ciudad Bolivar - Bogotá D.C.

El gobierno distrital de la ciudad de Bogotá D.C. se encuentra implementando actualmente una política pública que tiene como objeto la formulación y estructuración de una Red Distrital de Reconciliación. Con este proyecto el distrito busca lograr que los ciudadanos de Bogotá desarrollen y fortalezcan su integración por medio de acciones colectivas que refuercen las participación democrática y la apropiación de los principios de no violencia y derechos humanos con el propósito de consolidar acciones de respuesta ciudadana frente a los actos de violencia. Según este propósito, el Centro de Investigación sobre Dinámica Social de la Universidad Externado de Colombia realizó un proyecto piloto de análisis de las redes sociales (ARS) existentes en la localidad de Ciudad Bolívar.

\footnotetext{
${ }^{1}$ Leonardo Garavito G: luis.garavito@uexternado.edu.co , leonardogaravito@hotmail.com
} 
La escogencia de dicha localidad obedece a las condiciones sociales y múltiples expresiones de conflicto que se presentan en éste lugar. De hecho esta es una de las seis localidades declaradas en 'emergencia social' por parte de la administración distrital. En la localidad se encuentran entre 600.000 y 800.000 de habitantes ${ }^{2}$. En efecto, según un diagnóstico reciente (Secretaría de Gobierno Distrital, 2005) la mayoría de la población $(93.7 \%)$ se clasifica en los estratos socioeconómicos más bajos ( 1 y 2 ). Del total de hogares en la localidad un $25 \%$ es caracterizado en situación de indigencia y un 76\% (que incluye el anterior $25 \%$ ) está ubicado bajo la línea de pobreza.

De forma adicional, en Ciudad Bolívar se manifiestan diversas expresiones de conflicto que incluyen: conflictos asociados a la convivencia cotidiana (violencia intrafamiliar, pandillas y delincuencia común); conflictos asociados a la relación entre la localidad y el resto de la ciudad (manejo de residuos, pues allí se sitúa el relleno sanitario de Doña Juana que sirve a toda la ciudad, proyectos de explotación minera, infraestructural vial y servicios de transporte, entre otros); y por último, conflictos derivados del conflicto armado nacional, en tanto que se reconoce en su territorio la actividad de organizaciones armadas al margen de la ley.

Dado este contexto inicial, el ARS realizado ofrece un diagnóstico acerca del estado de las relaciones entre organizaciones de la sociedad civil en la localidad de Ciudad Bolívar, con respecto a la gestión compartida de acciones y proyectos. De una parte, se toma como definición básica para el concepto de 'organizaciones de la sociedad civil': un tipo de relación social que posee un equipo de trabajo comprometido en una actividad de tipo específico (Knoke \& Kaufman, 1990, 86). Acotto $(2003,37)$ precisa acerca del tipo de actividades que caracterizan a las organizaciones de la sociedad civil afirmando que tienden a dar respuestas a necesidades sociales grupales o colectivas. En particular, este tipo organizaciones no suele poseer límites rígidos en términos de los roles de sus miembros, o el mantenimiento de normas colectivas sostenidas por medios coercitivos tal cómo sucede en la mayoría de instituciones públicas o sociedades comerciales. En contraste, según Knoke (citado por Kaufman, 86) estas organizaciones son menos rígidas y poseen límites porosos definidos principalmente por sus miembros en sí, y sus líderes poseen poco poder sobre ellos.

2 Según proyecciones del Departamento Administrativo Nacional de Estadística -DANE- (2005) la
población en la localidad asciende a 713.762 individuos; y, según el Departamento Administrativo de 
De otra parte, la distinción entre acciones y proyectos ${ }^{3}$ como formas de gestión compartida se basa en el carácter más informal, de corto plazo o coyuntural de las acciones; y el acuerdo más formal, planeado y con mayor proyección temporal de los proyectos. De tal forma, este análisis pretende ser un aporte para la administración distrital en su interés de construcción de alianzas sociales que aporten a la consolidación de la red distrital de reconciliación.

Como punto de partida se encontró una presencia relevante de organizaciones trabajando en la localidad en múltiples temáticas (ver tabla a continuación); no obstante menos del $20 \%$ expresó compartir proyectos o acciones con otras organizaciones de la localidad. Según listados e inventarios de organizaciones facilitados por instituciones distritales y locales $^{4}$ se identificaron cerca de mil organizaciones presentes en la localidad, sin embargo el grupo central de organizaciones con las cuales se realizó el análisis convocó a 182.

\begin{tabular}{|l|c|c|}
\hline & Cantidad & Porcentaje \\
\hline Apoyo al desarrollo comunitario & 42 & 23.08 \\
\hline Apoyo al adulto mayor & 16 & 8.79 \\
\hline Mejorar calidad de vida de la población & 24 & 13.19 \\
\hline Promoción de la cultura & 35 & 19.23 \\
\hline Apoyo a grupos vulnerables & 7 & 3.85 \\
\hline Fomento de la actividad económica & 14 & 7.69 \\
\hline Promoción de valores & 27 & 14.84 \\
\hline Apoyo a la niñez & 17 & 9.34 \\
\hline
\end{tabular}

Tabla 1. Propósito de las organizaciones

A partir de los resultados principales del ARS se identificó que las redes de proyectos y acciones compartidas, en el período 2005-06, son inconexas y poco densas; en las cuales es posible encontrar, sin embargo, algunos componentes y núcleos que podrían llegar ser claves para el desarrollo de la conectividad de estas redes. Estos subgrupos confluyen, principalmente, en torno a temas como el fomento de actividades artísticas y culturales, la asistencia a grupos de población

Planeación Distrital (2003), encuesta de calidad de vida 2003, la población en Ciudad Bolívar son 602.697, la cual equivale al $10.4 \%$ del total de la población de la ciudad de Bogotá.

${ }^{3}$ La selección de estos dos tipos de vínculos se basa en su uso en previos proyectos de ARS: (Palacio et al, 2005) y (Palacio et al, 2003).

${ }^{4}$ A. Registro del programa de fortalecimiento al tejido local (SGD): 1117 referencias a organizaciones; $B$. Registro de la mesa local de cultura de Ciudad Bolívar: 397 referencias; C. Registro de la mesa local de juventud de Ciudad Bolívar: 102 referencias. 
vulnerable, o el desarrollo local. En relación a la conectividad de estas redes es relevante resaltar la participación de algunas organizaciones públicas y autoridades locales tales como la alcaldía local, la mesa local cultura, la de juventud, y varías juntas de acción comunal ${ }^{5}$. Una presentación detallada de estos resultados y el planteamiento de algunos puntos de reflexión acerca de las posibles tensiones entre la iniciativa política de crear una red social y la dinámica social que efectivamente se da entre las organizaciones en la localidad, son los objetivos principales del siguiente texto.

\section{Aspectos metodológicos}

¿Qué tantas organizaciones en la localidad de Ciudad Bolívar gestionaron proyectos y acciones compartidas con otras, en el periodo 2005-06? En relación a aquellas que lo hicieron ¿Estos vínculos estructuran redes sociales? ¿Cuáles son sus características? Y más allá ¿Un diagnóstico inicial al respecto, qué información aportaría a una política pública que favorezca el desarrollo de redes sociales en Bogotá? Las anteriores preguntas estructuran el ejercicio desarrollado a continuación.

Los tres primeros interrogantes se refieren directamente al ARS aplicado entre las organizaciones participantes. Por lo tanto, a continuación se presentan las medidas e indicadores seleccionados para la caracterización tanto de las redes en su totalidad, como de la participación de las organizaciones en estas. De otra parte, la última pregunta es abordada en la conclusión en tanto su carácter más propositivo en relación al aporte que el ARS planteado puede brindar a la política pública.

Sobre las organizaciones participantes, la definición de los actores para este ARS se ajustó al siguiente criterio: Organizaciones de la sociedad civil sin ánimo de lucro cuyo objeto de trabajo, cualquiera que fuese, se realizará en la localidad de Ciudad Bolívar, y que aceptaran participar en el ejercicio. Con las organizaciones que cumplieron esta condición se conformó un conjunto denominado 'grupo central'. En efecto, el grupo central quedó compuesto por 182 organizaciones. Entre los actores de este grupo se indagó acerca de sus vínculos de gestión compartida, con lo cual se identificó una red de proyectos y otra de acciones compartidas (2005-06).

\footnotetext{
${ }^{5}$ Según la ley 743 de 2002, que desarrolla lo referente a los organismos de acción comunal, una Junta de Acción Comunal -JAC- es una organización cívica, social y comunitaria de gestión social, sin animo de lucro, de naturaleza solidaria, con personería jurídica y patrimonio propio, integrada voluntariamente por los residentes de un lugar que aúnan esfuerzos y recursos para procurar un desarrollo integral, sostenible y sustentable con fundamento en el ejercicio de la democracia participativa.
} 
De forma complementaria, se estructuraron otras dos redes. Además del grupo central se construyó otro conjunto diferente de actores denominado 'entorno organizacional del grupo central'. El entorno organizacional se refiere otros actores, fuera del grupo central, vinculados a este por al menos uno de sus participantes. A diferencia del grupo central, la naturaleza de las organizaciones en el entorno es diversa: otras organizaciones adicionales cuyo espacio de acción no es la localidad de Ciudad Bolívar, organizaciones privadas, agencias internacionales, y entidades públicas del orden local, distrital y nacional. Así, el entorno organizacional de grupo central reúne otras 152 organizaciones adicionales. A partir de los vínculos de las organizaciones del grupo central con las del entorno se identificaron las redes de proyectos y acciones compartidas (2005-06).

El proceso de identificación del grupo central se basó en inventarios y directorios de organizaciones. En estos se detectaron 957 organizaciones; sin embargo, sólo 150 de estas llegaron a ser parte del grupo central. En los casos de las organizaciones descartadas, se encontró que muchas de estas habían cesado su actividad total o temporalmente, otras ya no trabajaban en la localidad, y en menor medida, no estaban interesadas en participar en el ejercicio. La segunda fuente para la identificación de otras organizaciones para el grupo central fue indagar entre la ya encontradas, sí conocían otras posibles participantes. Por medio de esta técnica se incluyeron 32 organizaciones adicionales. Así, finalmente, el grupo central quedó conformado por 182 actores $^{6}$ que participaron plenamente en la recolección de información acerca de la gestión de proyectos y acciones compartidas.

Sin embargo, no todas las 182 organizaciones del grupo central habían realizado acciones o proyectos compartidos en el período 2005-2006. Según lo anterior y con el fin de concentrar el diagnóstico en la dinámica relacional de las organizaciones que si se encontraban efectivamente vinculadas en el lapso temporal definido, se excluyeron del grupo central aquellas que estaban totalmente aisladas en cada una de las redes. Así el grupo central en general, sufrió una última variación en función a la delimitación temporal: en la red de proyectos compartidos el grupo central definitivo quedó constituido por 48 organizaciones, y en la red de acciones compartidas quedó conformado por 84.

\footnotetext{
6 Por solicitud expresa de algunas de las organizaciones participantes no se hará referencia a los nombres propios de las organizaciones participantes. Las personas interesadas en acceder al detalle de esta información deben establecer un contacto directo con el Centro de Investigación de Dinámica Social, de la Universidad Externado de Colombia.
} 
Los indicadores para la caracterización de las redes de proyectos y acciones, en su conjunto, fueron escogidos en función de los tipos de redes a observar: 1. El grupo central, y 2. El grupo central a través de su entorno organizacional. Por una parte, las redes del grupo central se refieren a redes de un modo, es decir que involucran medidas sobre un sólo grupo de actores (Wasserman and Faust, 1994, 36). Así, en el grupo central se analizaron los vínculos de proyectos y acciones compartidas de las organizaciones entre sí mismas. Por otra parte, las redes del grupo central a través de su entorno organizacional se refieren redes de dos modos, las cuales involucran medidas sobre dos grupos de actores (Wasserman and Faust, 38). En este sentido, en estas redes se analizaron los vínculos de las organizaciones de los grupos centrales con las organizaciones en su entorno.

Los indicadores seleccionados para la caracterización de las redes del grupo central son su tamaño, densidad y número de componentes. Estos buscan brindar un panorama inicial de las redes vistas como un todo. El tamaño se refiere a la cantidad de organizaciones participantes, la densidad a la cantidad de vínculos efectivos en comparación a los vínculos potenciales de de la red, y el número de componentes se refiere a la cantidad de subgrupos, desconectados entre si, que conforman las redes.

En segundo lugar, en tanto a la participación de los actores en las redes, se plantea un análisis de la distribución de su popularidad y expansión. El grado de popularidad se refiere a la cantidad de veces que un actor es escogido por los otros para ser parte de la red. En términos de las redes observadas, se refiere a cuántas veces una organización es reconocida por otras como su compañera en la realización de acciones o proyectos compartidos. Entonces, la organización más popular será aquella que haya sido la más nombrada por el resto. En contraste, el grado de expansión tiene en cuenta la cantidad de vínculos que declara tener cada organización. Así, el grado de expansión corresponde al número de organizaciones que cada actor identifica como sus vinculadas. Entre más vínculos declaré una organización, mayor será su grado de expansión en la red.

De forma seguida se abordan los núcleos de las redes del grupo central. Esta parte ofrece una mirada de las redes a algunos de sus subgrupos más relevantes en la red. Los núcleos se refieren a subgrupos de actores, donde sus participantes comparten un grado mínimo de cohesión. El objetivo de identificar estos núcleos es, por una parte, hacer visible la presencia de subgrupos que ocupan una posición estructural relevante y potencial en relación a la conectividad general de las redes analizadas; y, por otra, ofrecer ejemplos acerca de cómo es posible identificar 
grupos de organizaciones, los cuales pueden ser útiles para focalizar intervenciones en términos de política pública que pretendan generar efectos de conectividad en la red. Así, en el núcleo de la red de acciones y de proyectos se observan tanto los indicadores globales planteados anteriormente, como la distribución del grado de popularidad y expansión de las organizaciones que los componen.

De igual manera que para el grupo central, las redes relacionadas con el entorno organizacional son presentadas primero a través de las medidas globales de tamaño y número de componentes. No obstante, los indicadores acerca de la participación de los actores en la red difieren dada su condición de red de dos modos. Esto se debe a que, como es propio de las redes de dos modos, sus vínculos son unidireccionales. Es decir que se extienden desde las organizaciones del grupo central hacia las organizaciones del entorno, sin embargo, no se cuenta con información por parte de las organizaciones del entorno organizacional, y por tanto, los vínculos no pueden regresar hacia el grupo central. En este sentido las organizaciones del entorno son diferentes a las del grupo central, por lo cual no son vistas como actores propiamente dichos, sino como eventos a los cuales se afilian los actores del grupo central. Por tanto, las propiedades de los actores que son observadas en una red de dos modos no son la popularidad y la expansión, sino: las tasas de participación y el tamaño de los eventos. Por un lado, las tasas de participación son el número de eventos con los cuales cada actor está afiliado (Wasserman \& Faust, 312). Para estas redes, la tasa de participación es propiedad de los actores del grupo central, en función del número de organizaciones del entorno con las cuales se encuentran afiliadas. Por el otro, el tamaño de los eventos es dado por el número de actores afiliados a cada evento (Wasserman \& Faust, 312). Es decir que, el tamaño es una propiedad de las organizaciones del entorno, e indica cuántas organizaciones del núcleo se afilian con cada una de ellas.

Por último, en la sección de conclusiones se retoma el tema del aporte de información que un ARS le puede brindar a una política pública que favorezca el desarrollo de redes sociales en Bogotá. En un sentido amplio, se afirma que el ARS ofrece una herramienta potencial para el análisis de los procesos de políticas públicas (formulación, gestión y evaluación), en tanto a la participación y relevancia creciente que las organizaciones de la sociedad civil en estos. Según Villar (2003, 13) las organizaciones de la sociedad civil han logrado incorporar nuevos temas en la agenda pública, han promovido nuevas voces y diversos puntos de vista para el debate de las políticas. No obstante, cabe tener en cuenta la advertencia de evitar la idealización de sus posibles acciones, de observarlas como la salida viable a la 
profunda crisis social y política que invade nuestras vidas (Acotto, 13) (en referencia a Latinoamérica), para enfocarse más en un análisis de sus posibilidades reales.

Así las cosas, a continuación se presentan un conjunto de redes sociales acerca de la dinámica relacional entre organizaciones en la localidad de Ciudad Bolívar, en relación a su gestión de proyectos y acciones compartidas en el periodo 2005-2006.

\section{Las redes de proyectos y acciones compartidas entre organizaciones en la localidad de Ciudad Bolívar, 2005-2006 \\ 2.1. Las redes del grupo central}

Para comenzar, en relación a las medidas globales de las redes del grupo central, se encuentra que la red de acciones compartidas es de mayor tamaño que la de proyectos, sin embargo la red proyectos presenta una mayor densidad que la de acciones. Así las cosas, con respecto su tamaño, la red acciones compartidas agrupa a 84 organizaciones mientras que la de proyectos cuenta con 48 . En contraste, en tanto a la densidad de cada red, es decir a la proporción de vínculos que efectivamente existen en relación a todos los vínculos posibles, la red de proyectos compartidos presenta una densidad del $2.1 \%$ frente al $1.2 \%$ en la red de acciones.

A pesar de la diferencia en su densidad ambas redes tienden a ser relativamente frágiles, ya que su potencialidad para conectar a las organizaciones involucradas es utilizada de forma mínima. Redes poco densas como estas, enfrentan un riesgo latente de desintegrarse, dado el caso en que los vínculos existentes entre las organizaciones se agoten y no se creen otros nuevos. En este sentido, para estas redes cada vínculo existente o nuevo es un aporte relevante a su densificación. Por lo tanto estimular la creación y el desarrollo de vínculos de gestión compartida entre las organizaciones participantes sería un objetivo difícil de ignorar en una política pública dirigida al desarrollo de redes sociales como estas.

A continuación se presentan los grafos de las redes: 


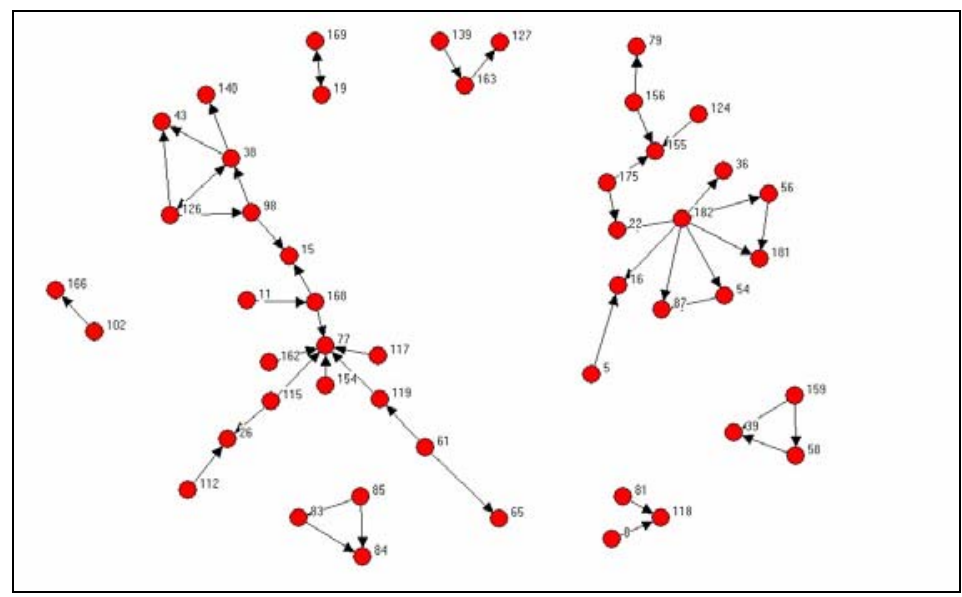

Figura 1. Red de proyectos compartidos 2005-2006, grupo central ${ }^{7}$.

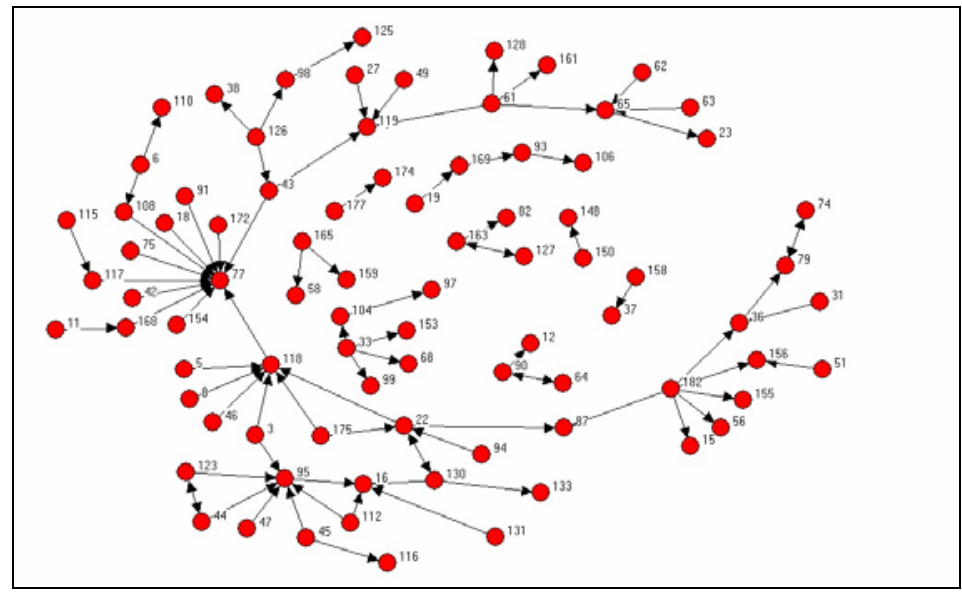

Figura 2. Red de acciones compartidas 2005-2006, grupo central.

De otra parte, como se puede observar en los grafos, tanto la red de proyectos como la de acciones compartidas son redes inconexas. Es decir, poseen menos vínculos que el mínimo para que cualquier par de actores se puedan conectar entre sí; en contraste, cada organización social se encuentra vinculada a un subgrupo y aislada del resto. Cada subgrupo de organizaciones corresponde a un componente de la red. A continuación se describen las redes de proyectos y acciones compartidas a través de sus componentes.

\footnotetext{
${ }^{7}$ Todas las gráficas son realizadas en UCINET 6.0
} 
En primer lugar, en la red proyectos compartidos se identifican 8 componentes: dos principales que agrupan la mayoría de las organizaciones (uno 18 y otro 14), 4 componentes más con 3 organizaciones cada uno, y 2 componentes adicionales con 2 organizaciones. Para un total de 48 organizaciones en 8 componentes. El componente principal (18) de la red de proyectos está conformado en su mayoría, de organizaciones comunitarias del orden más local (Juntas de Acción Comunal) vinculadas entre sí, y con algunas otras organizaciones con diferentes propósitos (atención a grupos de población vulnerable o calidad de vida). El segundo componente (14) integra principalmente organizaciones que promueven actividades artísticas y culturales. El resto de componentes corresponde a 4 tríos y 2 parejas en dónde se encuentran otras varias Juntas de Acción Comunal y organizaciones culturales.

En segundo lugar, en la red de acciones compartidas se identifican 9 componentes: uno que agrupa la mayor parte de actores (59), otro con 6 actores, el siguiente con 4, 3 componentes más con 3 actores cada uno, y 2 componentes más con 2 organizaciones cada una. Para un total de 84 organizaciones en 9 componentes. El componente principal de esta red, con 59 actores, es un caso especial. Aunque aparentemente agrupa a número grande de organizaciones, en comparación a los otros componentes, sus vínculos son frágiles entre si y más bien reúne en su interior varios subgrupos que incluyen autoridades locales públicas, juntas de acción de comunal, organizaciones culturales y artísticas, entre otras. Esto es evidente en tanto que la conexión en este componente depende en muchos casos de un actor y sus vínculos que, de no estar presentes, lo fragmentarían en dos o más subgrupos. Esta situación se puede observar en el siguiente grafo, en el cual se resalta en color rojo a aquellos actores que configuran los puntos de corte potenciales en dicho componente. 


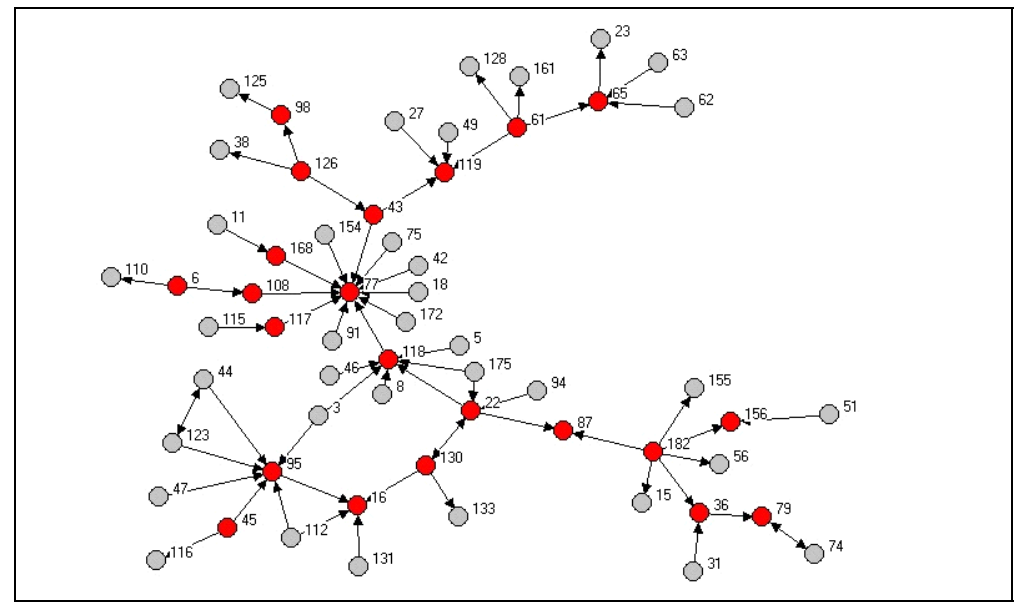

Figura 3. Componente principal de la red acciones compartidas (2005-2006).

Los nodos en color rojo representan los puntos de corte dentro de la red.

En relación al resto de componentes de la red de acciones compartidas, se identifica una situación similar al caso de la red de proyectos. Es decir, son subgrupos pequeños, tríos o parejas en los cuales se encuentran otras organizacionales similares: autoridades locales, Juntas de Acción Comunal, organizaciones artísticas y culturales, de fomento al desarrollo local y de atención a grupos de población vulnerable.

\subsubsection{Popularidad y expansión de las organizaciones en la red.}

Tanto en la red de acciones como en la de proyectos compartidos la participación de los actores tienden a ser bastante heterogénea. Esta participación puede caracterizarse a través de los grados de popularidad y expansión de los actores en la red. Que sus posiciones sean heterogéneas conlleva a que la participación de las organizaciones varía de forma relevante entre unas y otras. Es decir, al observar las organizaciones según sus grados de popularidad y de expansión en la red, se encuentran unas pocas organizaciones con altos grados de una u otra condición, y de resto se identifica con bajos grados a la mayoría de participantes. 


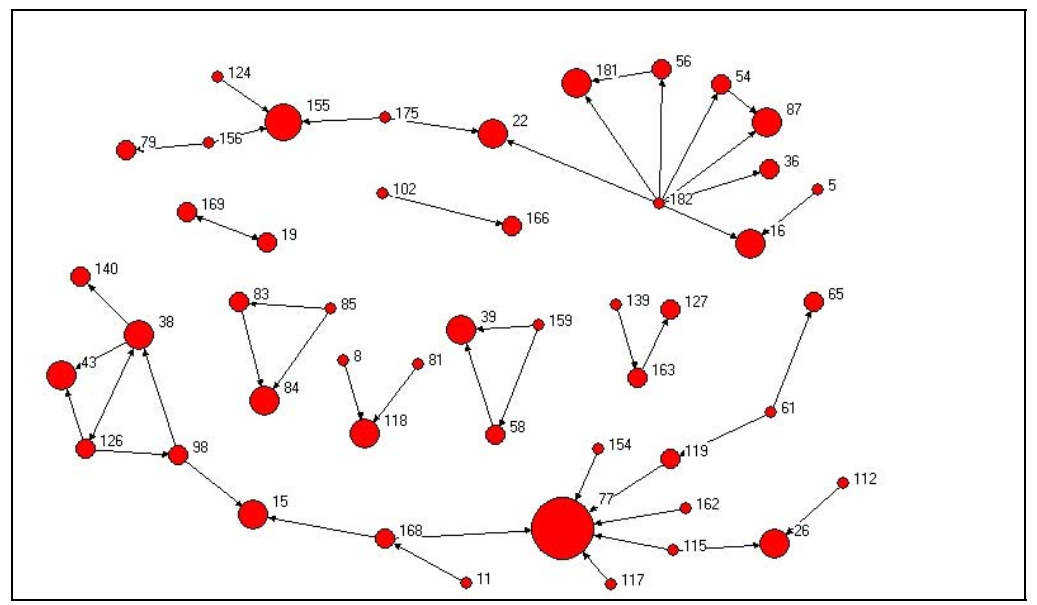

Figura 4. Red de proyectos compartidos 2005-2006, según grado de popularidad.

El tamaño de los nodos varía de acuerdo al grado de popularidad de los actores, los más grandes son los más populares.

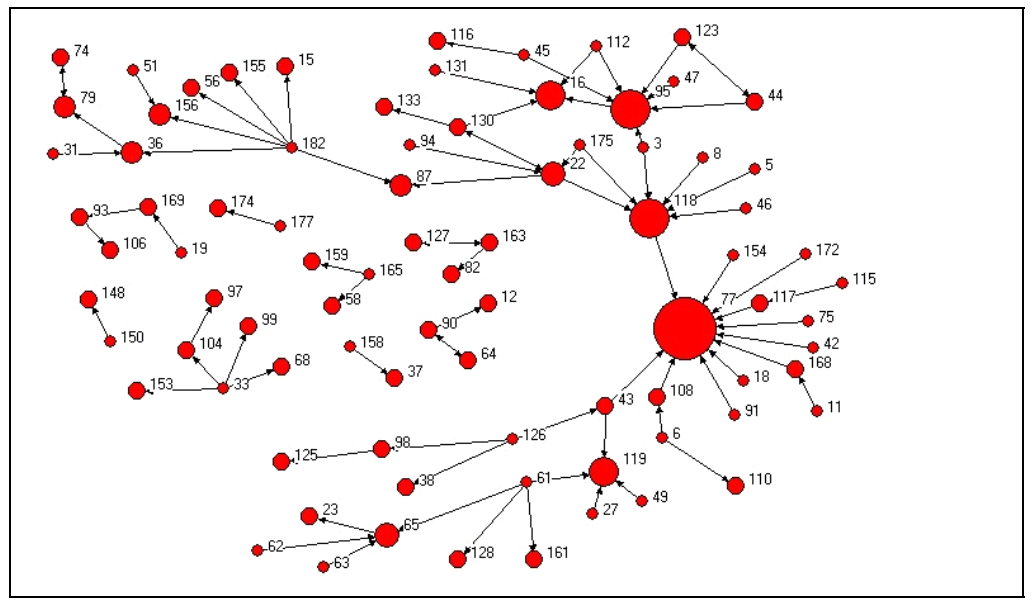

Figura 5. Red de acciones compartidas 2005-2006, según grado de popularidad.

El tamaño de los nodos varía de acuerdo al grado de popularidad de los actores, los más grandes son los más populares.

En primer lugar, en tanto a la popularidad, en ambas redes se encuentran unas pocas organizaciones con grados muy superiores al promedio. En ambas redes el valor promedio del grado de popularidad para cada actor es 1, es decir, que cada organización es nombrada como vinculada a otras, en promedio, una vez. Sin embargo la distribución de esta popularidad es bastante inequitativa en cada una de las redes. De una parte, en la red proyectos se encuentra que el grado máximo de popularidad es 6 y sólo lo tiene un actor de la red; mientras que el mínimo es 0 , y este es el grado de popularidad de 18 de las organizaciones participantes. De otra parte, y más inequitativo aún, en la red de acciones se evidencia que el máximo grado de popularidad de 11 para una sola organización, en contraste, con un grado mínimo de 0 , para 34 organizaciones. 
En particular, es interesante resaltar el caso de los vínculos de la organización No. 77 que corresponde a la Asociación de Usuarios de Hospital Vista Hermosa. Esta presenta uno de los grados mayores de popularidad, pero uno de los grados menores de expansión (ver gráficas siguientes). En este caso cabe aclarar que dicha organización declara que su propósito principal es promover la participación de los usuarios de la salud a los programas de prevención y promoción. En este sentido es posible que su enfoque hacia el usuario final desplace el reconocimiento de los vínculos que posee con otras organizaciones sociales, y por esto existe una gran disparidad entre su grado de popularidad y de expansión.

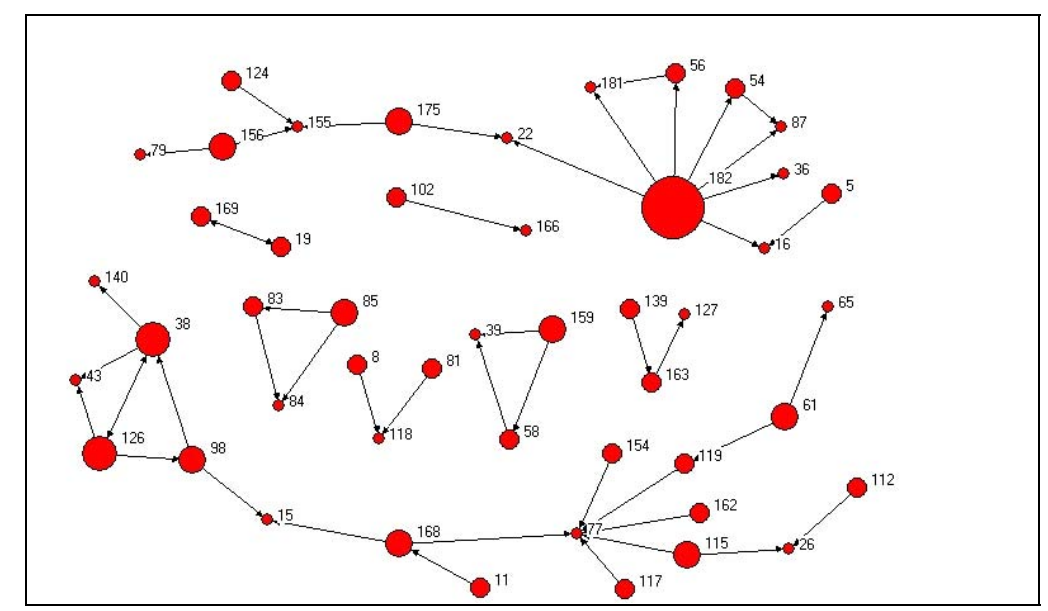

Figura 6. Red de proyectos compartidos 2005-2006, según grado de expansión.

El tamaño de los nodos varía de acuerdo al grado de expansión de los actores, los más grandes son los más expansivos. 


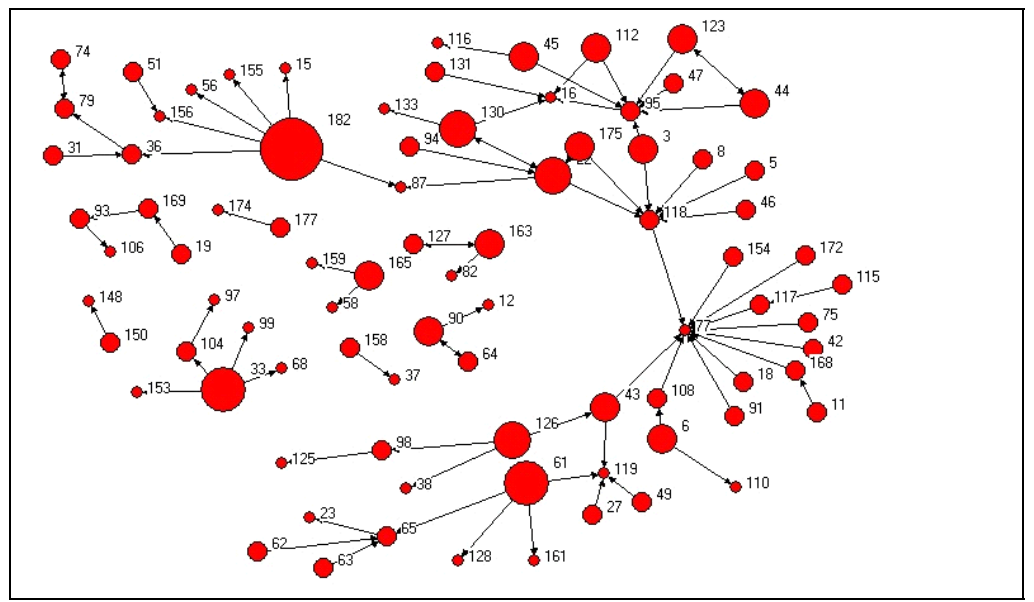

Figura 7. Red de acciones compartidas 2005-2006, según grado de expansión.

El tamaño de los nodos varía de acuerdo al grado de expansión de los actores, los más grandes son los más expansivos.

En segundo lugar, el grado de expansión de los actores en ambas redes también tiende a distribuirse de forma heterogénea. El promedio del grado de expansión en ambas redes es 1 , es decir que, en promedio, cada organización nombra a otra como vinculada a su gestión de proyectos o acciones compartidas. En la red de proyectos compartidos, el grado máximo de expansión es 7 y lo tiene sólo una organización; en oposición el grado mínimo de expansión es 0 y agrupa a 18 organizaciones. De forma un poco menos acentuada, en la red de acciones se observa un grado máximo de expansión de 6 , para un solo participante; y un grado mínimo de 0 para 28 de los participantes.

De acuerdo a la heterogeneidad en la distribución de la popularidad y la expansión en la redes de proyectos y acciones, se puede afirmar que la participación de la organizaciones en las redes es muy diferenciada. En unos pocos casos se evidencian organizaciones muy populares o expansivas en las redes, en comparación a muchas otras con muy pocos o incluso sólo un vínculo. En este sentido, desde el punto de vista de la popularidad y la expansión se corrobora que estas redes poseen una estructura relativamente frágil; en las cuales, si se retiraran aquellas organizaciones que tienen un grado de popularidad 0 de expansión menor a 3, ambas redes se desintegrarían.

Otro caso particular interesante es el del actor No. 182, el cual presenta un comportamiento inverso al del actor No. 77 (planteado anteriormente). Es decir, presenta uno de los mayores grados de expansión pero uno de los menores de popularidad. El actor No. 182 corresponde al Consejo Local de Cultura. Este caso es más complicado de explicar en tanto que su alto grado de expansión se entiende en 
términos de su condición de autoridad pública y de sus posibilidades de gestionar recursos públicos para el apoyo de acciones o proyectos de otras organizaciones. No obstante no es claro el por qué de su bajo grado de popularidad. A manera de hipótesis se propone que, ya que en la entrevista se preguntó acerca de los vínculos con otras organizaciones, los entrevistados que tenían vínculos con el actor No. 182 no los nombraron en tanto que no la perciben como una organización de la sociedad civil sino más como una institución pública de carácter local.

\subsubsection{El núcleo de las redes}

A pesar de la condición de fragilidad relativa de las redes observadas, es posible identificar, en estas, núcleos potenciales para su desarrollo. Los núcleos se refieren a estructuras al interior de la redes, en las cuales se encuentra un subgrupo de actores que cumplen con un grado mínimo de cohesión entre si. En este caso el grado mínimo de cohesión de cada actor del núcleo se refiere a contar con al menos dos vínculos, ya sea que estos partan desde el actor o sean recibidos por él, o uno recibido y el otro dado. Sin embargo, cabe resaltar que el tipo de núcleos identificados obedecen a aquellos con el menor grado de cohesión posible, lo cual sigue corroborando la tendencia a un grado de conectividad bajo en estas redes.

Aplicando el método de suprimir de forma progresiva los actores y los vínculos más periféricos en cada una de las redes, hasta encontrar estructuras que no son susceptibles de ser reducidas, se identificó el núcleo en cada red. En un sentido metafórico estos son el corazón de cada red, subgrupos con potencialidad relevante para estructurar al resto del conjunto en tanto que conforman configuraciones irreducibles. 


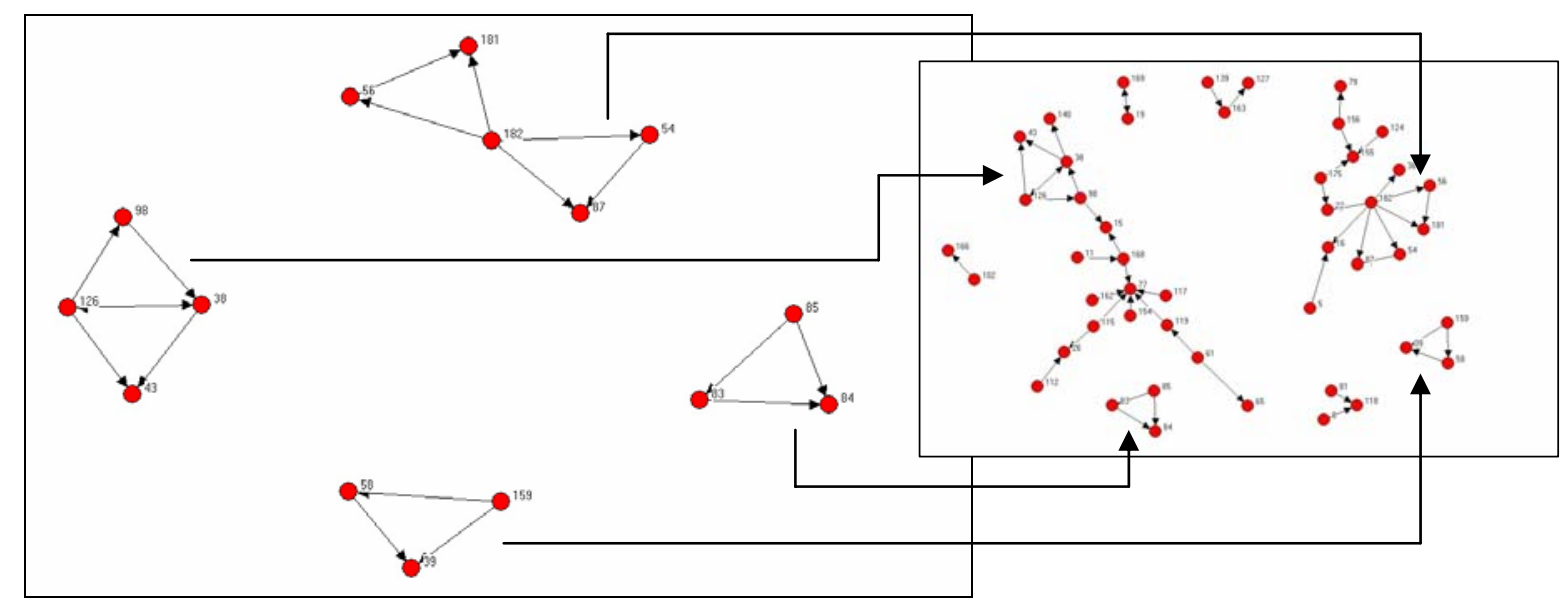

Figura 8. Núcleo de la red de proyectos compartidos 2005-2006.

En tanto al núcleo de la red proyectos compartidos, al igual que la red en su globalidad, es inconexo, y está conformado por cuatro componentes que agrupan un total de 15 organizaciones. El grado de densidad del núcleo es del 8.6\%, y aunque es mayor que el de la red completa ( $2.1 \%$ ), es relativamente bajo teniendo en cuenta que al observar el núcleo se está enfocando una de sus secciones más densas.

La participación de las organizaciones en el núcleo de la red de proyectos compartidos, en términos de sus grados de popularidad y expansión, evidencia una distribución un poco menos inequitativa en comparación a la red de proyectos como totalidad. Sin embargo, para ser el corazón de la red, aún tiende a ser bastante heterogénea. En promedio cada organización en el núcleo tiene un grado popularidad y de expansión de 1.2, apenas un poco más que el promedio en la red de proyectos completa (grado 1). Al comparar la distribución del grado de expansión con la del de popularidad se encuentra que el primero se distribuye de forma más heterogénea que el segundo. En términos del grado de expansión el máximo es 4 y corresponde a una sola organización, mientras que el mínimo es 0 y agrupa a 4 de las 15 organizaciones en el núcleo. En tanto al grado de popularidad el máximo es 2 y es compartido por 6 organizaciones, y el mínimo es de 0 para solo una organización.

El hecho de que el grado de expansión se encuentre distribuido de forma más heterogénea que el de popularidad, en el núcleo de la red proyectos compartidos, indica que la participación de algunas de las organizaciones depende de haber sido reconocidas por otras, es decir por su popularidad, y no por haber declarado sus 
propios vínculos, su expansión. En este sentido cabe resaltar que las organizaciones más expansivas del núcleo son, en mayor medida, las responsables de la cohesión del mismo. Al identificar cuál es el carácter de las organizaciones más expansivas en este núcleo se confirma el importante rol de autoridades locales del tipo de las Juntas de Acción Comunal y el Consejo Local de Cultura.

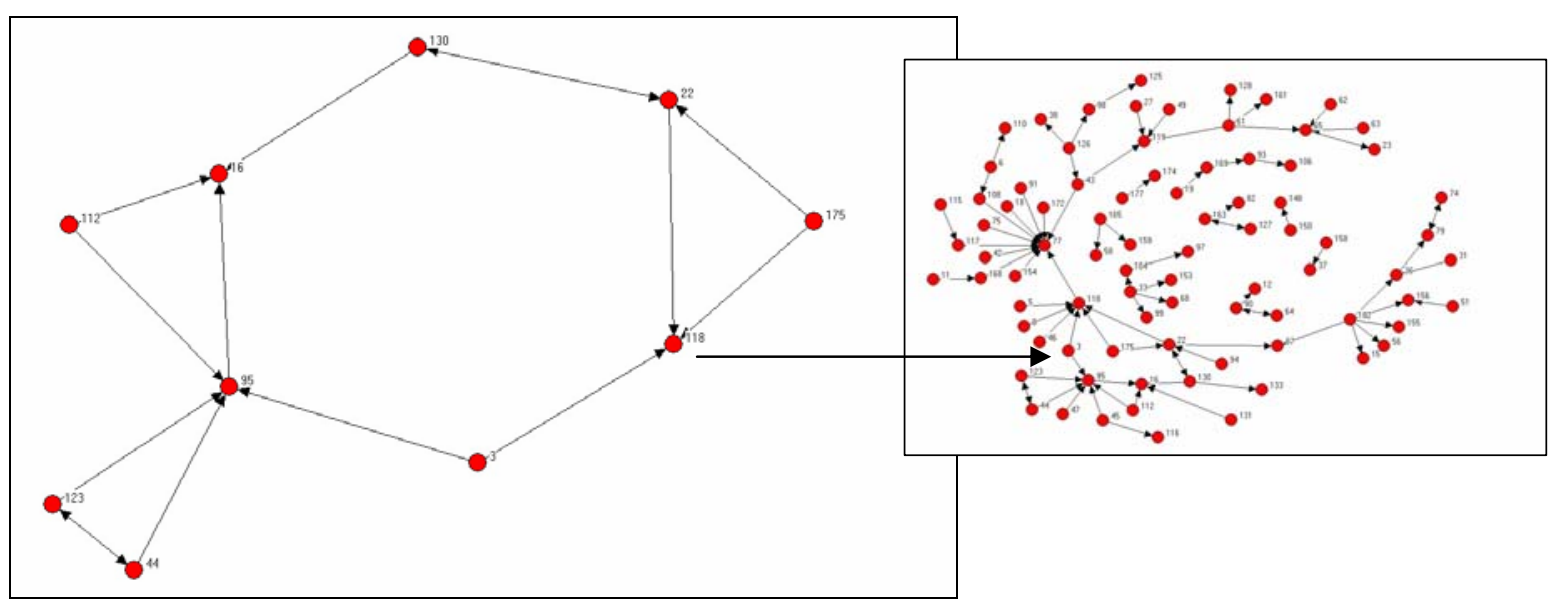

Figura 9. Núcleo de la red acciones compartidas 2005-2006.

En contraste, el núcleo de la red de acciones compartidas 2005-2006 corresponde a un componente único, que agrupa un total de 10 organizaciones. La densidad de esta estructura es del $16.7 \%$, aproximadamente el doble de densidad del núcleo de la red de proyectos ( $8.6 \%)$, y casi 14 veces más densa que la red de acciones compartidas en su totalidad (1.2\%). No obstante para ser un núcleo también se puede considerar con una densidad mayor pero relativamente baja aún.

La distribución de la popularidad y la expansión en este núcleo es menos inequitativa en comparación a su distribución en la red de acciones compartidas en general. No obstante, al igual que el núcleo de la red proyectos, no pierde su tendencia a la heterogeneidad. En promedio la popularidad y la expansión presenta un grado de 1.5 , un medio más que el promedio para la totalidad de la red de acciones (grado 1). En comparación al núcleo de la red de proyectos, la distribución de los grados expansión y popularidad en la red de acciones está más balanceada, pero en este caso la distribución de la popularidad es un poco más inequitativa que el de la expansión. De hecho, en tanto a la distribución de la expansión se encuentra un grado máximo de 2 el cual aplica para 7 de las 10 organizaciones, y un mínimo de 0 en para dos organizaciones. Respecto a la popularidad se observa 
un grado máximo de 4 para una organización y un mínimo de 0 para 3 organizaciones más.

Que el grado de expansión sea menos inequitativo que el de popularidad, en el núcleo de la red de acciones compartidas 2005-2006, indica que la participación de las organizaciones en el mismo depende más de los vínculos declarados por cada actor, que por del hecho de ser nombrados por otros. En este sentido cabe resaltar que el tipo de organizaciones que participan en este núcleo se caracteriza por ser en su mayoría organizaciones que favorecen actividades artísticas y culturales. De forma más o menos similar al núcleo de la red de proyectos compartidos, se identifica la presencia de una autoridad local como es el Consejo Local de Juventud y una Junta de Acción Comunal adicional.

Al comparar las redes totales y sus núcleos se evidencia que algunas de las características generales de las redes se reflejan de forma más o menos similar, pero no idéntica, en sus núcleos. En primer lugar, la tendencia a un nivel bajo de densidad es una característica común a ambas redes y a sus núcleos. No obstante, cabe resaltar que mientras la red de proyectos es relativamente más densa que la de acciones, en contraste, el núcleo de la red de acciones es más denso el de la red de proyectos. En este mismo sentido, la tendencia a un alto nivel de heterogeneidad en la distribución de la expansión y la popularidad de las organizaciones en la redes, también se reproduce, en menor medida, en sus núcleos. No obstante, vale la pena resaltar que en el núcleo de la red acciones compartidas se encuentra un poco más cohesionado que el de la red proyectos; lo cual puede tomarse como una señal de que las organizaciones prefieren o les es más sencillo optar por realizar acciones que proyectos compartidos. Esta preferencia o facilidad se tiende a confirmar al observar que el tamaño de la red de acciones compartidas es mayor en comparación al de la de proyectos.

\subsection{Las redes a través de su entorno organizacional}

El entorno organizacional es una fuente potencial de vínculos indirectos entre las organizaciones participantes. El entorno organizacional está compuesto por un conjunto de organizaciones, que sin pertenecer directamente al núcleo de la red se encuentran conectadas con al menos uno de sus participantes. A diferencia del núcleo de las redes, la naturaleza de las organizaciones en el entorno es diversa, y entre estas se pueden identificar distintos tipos: organizaciones adicionales cuyo espacio de acción no necesariamente es la localidad de Ciudad Bolívar (49 en el entorno de la red proyectos compartidos y 63 en la acciones), algunas 
organizaciones de carácter privado ( 13 y 20 , en cada red de forma respectiva), unas pocas agencias internacionales ( 5 y 2 ), pero sobre todo organizaciones públicas del orden local ( 17 y 20), nacional ${ }^{8}$ ( 6 y 5) y distrital ( 21 y 12). Teniendo en cuenta lo anterior, a continuación se ofrece, primero, una descripción general de algunas de las características de las redes de proyectos y acciones, en su globalidad, vistas a través de su entorno; segundo, un acercamiento a la participación de las organizaciones participantes en la red; y tercero, una observación más detallada por medio de los núcleos presentes cada una de las redes.

En primer lugar, según su tamaño, la red de acciones compartidas incluyendo a su entorno organizacional, agrupa un mayor número de organizaciones que la red de proyectos. Por una parte, en la red acciones compartidas y su entorno se encuentran 204 organizaciones, de las cuales 82 (40\%) hacen parte del núcleo de la red y $122(60 \%)$ del entorno. Por otra, en la red de proyectos compartidos y su entorno se reúnen 183 organizaciones, entre ellas, 72 (39\%) en el núcleo y 111 $(61 \%)$ en el entorno. Por lo tanto, a pesar de su diferencia de tamaño, en términos relativos presentan una relación similar entre el número de organizaciones presentes tanto en el grupo central como en el entorno. De manera adicional, de igual forma que para las redes del grupo central, la red de acciones compartidas a través del entorno agrupa una mayor cantidad de organizaciones que la de proyectos.

En segundo lugar, de acuerdo a su composición, ambas redes son inconexas en un alto grado. Observadas a través de su entorno tanto la red de acciones como de proyectos compartidos se encuentran divididas en múltiples componentes, 41 en la red de acciones y 36 en la red proyectos. En este sentido el mayor tamaño de la red de acciones se ve correspondido con un número también mayor de componentes. Señal que indica que no necesariamente la red más grande equivale a la red más conectada. Tal cantidad de componentes en cada una de las redes es algo que no se puede distinguir fácilmente a simple vista dada la cantidad de organizaciones participantes en el grupo central y en el entorno, sin embargo, como una ilustración general a continuación se presentan los grafos respectivos a cada caso:

\footnotetext{
${ }^{8}$ Esta categoría incluye algunas organizaciones del orden regional. Como por ejemplo la Gobernación de Cundinamarca o la Corporación Autónoma Regional (CAR).
} 


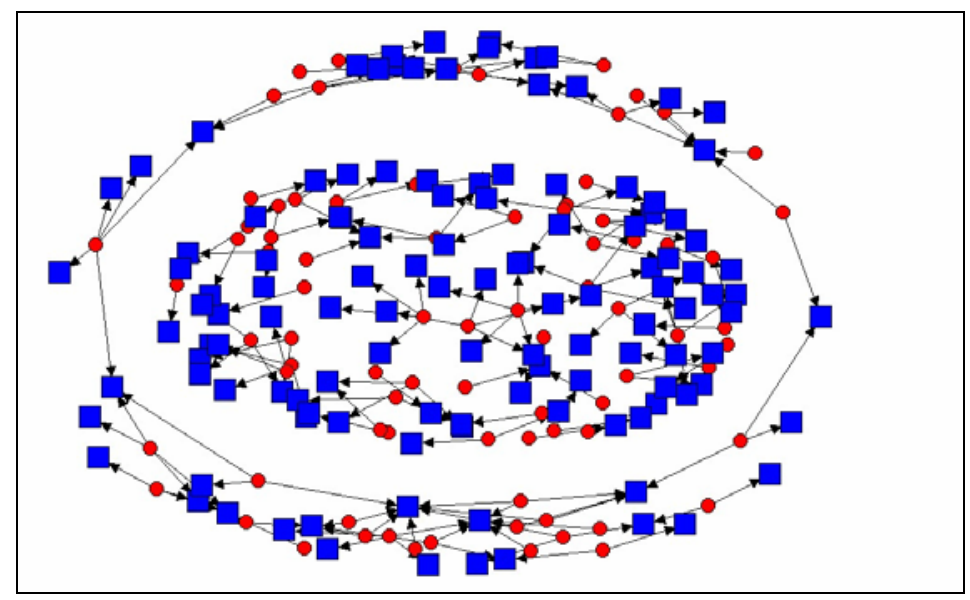

Figura 10. Red de acciones compartidas 2005-2006, a través de su entorno organizacional. Los círculos rojos son las organizaciones de la red, y los cuadros azules las organizaciones del entorno.

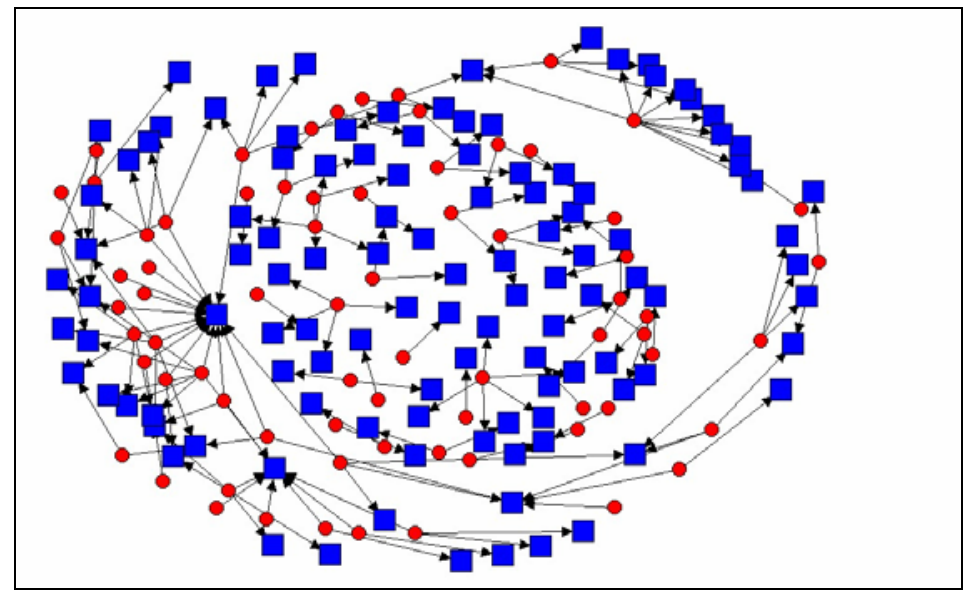

Figura 11. Red de proyectos compartidos 2005-2006, a través de su entorno organizacional. Los círculos rojos son las organizaciones de la red, y los cuadros azules las organizaciones del entorno.

En tercer lugar, el análisis de la participación de las organizaciones en las redes es realizada por medio de los dos siguientes indicadores: 1. La tasa de participación de las organizaciones del grupo central en el entorno, es decir el número de vínculos que declara cada organización del grupo central en relación a otras del entorno, y 2. El tamaño de las organizaciones del entorno, entendido como el número de veces que cada organización del entorno es nombrada por otras del grupo central. La diferencia entre estos indicadores y los grados de expansión y popularidad, radica en que esta red sólo tienen en cuenta los vínculos unidireccionales que parten de las organizaciones del grupo central hacia las del entorno, pero deja de lado tanto los vínculos de las organizaciones del grupo central entre si mismas, 
como posibles vínculos desde las organizaciones del entorno hacia las del grupo central.

A la luz de estos indicadores se observa, para comenzar, que la distribución del tamaño de las organizaciones del entorno en ambas redes es heterogénea. Esto significa que hay unas pocas organizaciones del entorno que tienden a un gran tamaño y muchas otras que tienen un tamaño que tiende a ser pequeño. El tamaño promedio de la organizaciones del entorno de la red de acciones compartidas es de 1.4, mientras que el de las de la red de proyectos es de 1.5. El tamaño máximo en la red acciones corresponde a una organización con tamaño grado 7 , y en la de proyectos a una grado 15. El tamaño mínimo corresponde en la red de acciones a 97 organizaciones del entorno con tamaño grado 1 y en la de proyectos con 88 grado 1.

En contraste con el tamaño de las organizaciones del entorno, la distribución de la tasa de participación de las organizaciones del grupo central es un poco menos heterogénea. Es decir que no se encuentra una diferencia tan acentuada entre las organizaciones del grupo central tienen más y menos vínculos con el entorno en ambas redes. La tasa de participación promedio para las organizaciones del grupo central de la red de acciones es 2, y para la red de proyectos es 2.2. En relación a las tasas de participación máximas en la red de acciones se encuentran 3 organizaciones con grado 5 , en la de proyectos una con grado 9. Respecto a las tasas mínimas de participación del grupo central en la red de acciones se encuentran 33 organizaciones grado 1 y en la red de proyectos 30 grado 1 .

Según los anteriores indicadores es posible afirmar que, en ambas redes, el tamaño de las organizaciones del entorno es más desigual que la participación de las organizaciones del grupo central. Esto implica que las organizaciones del grupo central tienden a una cantidad de vínculos más o menos uniforme con las del entorno; sin embargo, entre las organizaciones del entorno se podrían identificar algunas que son mucho más reconocidas que otras, por parte de las organizaciones del grupo central. Al identificar qué tipo de organizaciones son estas se encuentra que en su mayoría son de carácter público: p.e. tanto la Alcaldía Local de Ciudad Bolívar como el Instituto Distrital de Recreación y Deporte son las dos organizaciones del entorno con mayor tamaño en ambas redes. El carácter público de dichas organizaciones puede ser un punto interesante para la formulación de una política pública de redes sociales en Ciudad Bolívar; pues de acuerdo a los resultados anteriores, si estas organizaciones coordinan esfuerzos para la creación 
y desarrollo de tal red podrían estimular la gestión de acciones y proyectos entre las organizaciones de la localidad.

De forma adicional, aunque ambas redes presentan valores muy similares en los indicadores planteados, vale la pena resaltar que en la red proyectos compartidos, a través del entorno, es mayor tanto el tamaño promedio de las organizaciones del entorno como la tasa promedio de participación de las organizaciones del grupo central. Esto indica una mayor conectividad en la red de proyectos que en la de acciones compartidas $y$, de nuevo, ratifica que la red más grande no es necesariamente la más conectada.

En cuarto lugar, para análisis más detallado del efecto que tienen las organizaciones del entorno sobre el grupo central, se presentan a continuación los núcleos en cada una de las redes. De igual manera que para los núcleos de las redes del grupo central, se excluyeron de forma progresiva las organizaciones y vínculos más periféricos hasta encontrar los núcleos que ya no pueden ser reducidos. En este mismo sentido, el grado de cohesión mínimo también corresponde a por lo menos 2 vínculos recibidos para las organizaciones del entorno o de al menos 2 emitidos para las del grupo central.

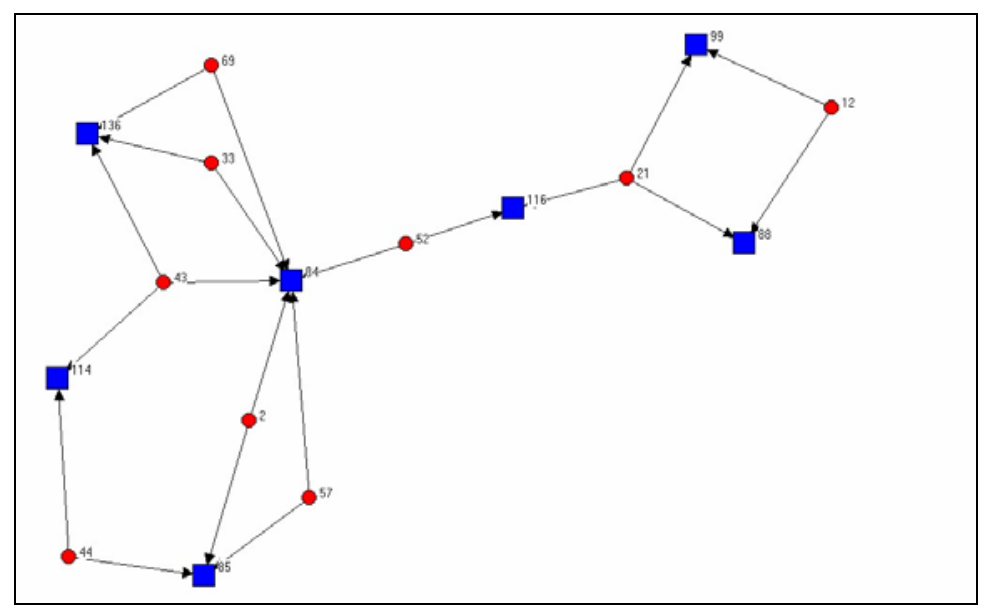

Figura 12. Núcleo de la red de acciones compartidas 2005-2006, a través de su entorno. Los círculos rojos son las organizaciones del núcleo de la red, y los cuadros azules las organizaciones del entorno. 


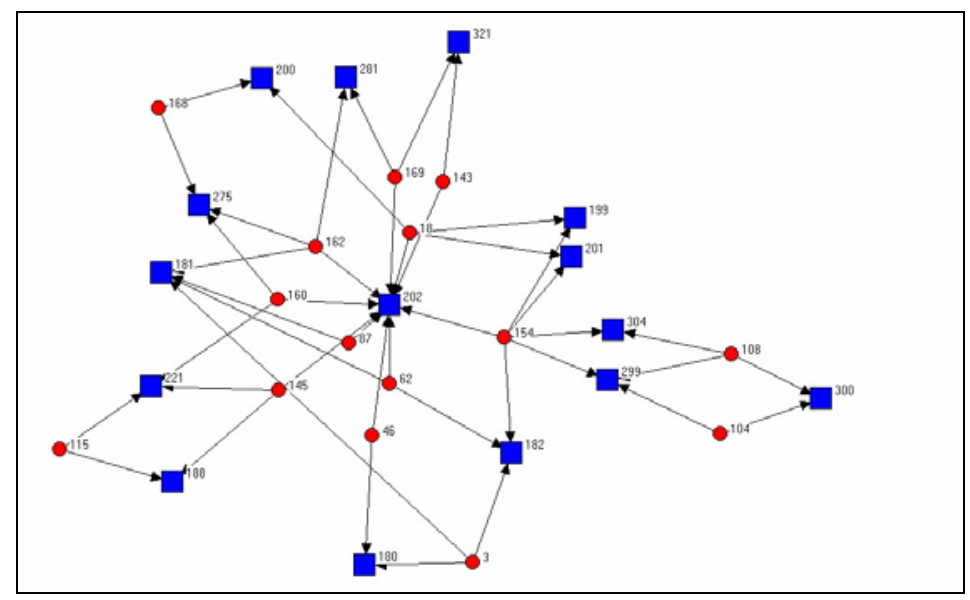

Figura 13. Núcleo de la red de proyectos compartidos 2005-2006, a través del entorno.

Los círculos rojos son las organizaciones del núcleo de la red, y los cuadros azules las organizaciones del entorno.

Por una parte, el núcleo de la red de acciones compartidas, a través del entorno, reúne 16 organizaciones, de las cuales $9(56 \%)$ son del grupo central de la red y 7 (44\%) del entorno. Al observar la tasa de participación de las organizaciones del grupo central del núcleo, se identifica una tendencia a la homogeneidad. Así, se encuentra que 2 organizaciones tienen una participación grado 3 y las restantes 5 grado 2. En contraste, la distribución del tamaño entre las organizaciones del entorno es mucho menos homogénea: una organización tiene tamaño grado 6 , seguida de dos más grado 3 , y la 4 restantes grado 2 .

Por otra parte, el núcleo de la red de proyectos compartidos, a través del entorno, integra 30 organizaciones, 15 (50\%) son del grupo central y 15 (50\%) del entorno. Según la distribución de la tasa de participación de las organizaciones del grupo central en el núcleo se encuentra una organización con participación grado 6, 2 más con grado 4, 6 de grado 3 y las 6 restantes con grado 2. Menos homogénea es la distribución del tamaño entre las organizaciones del entorno, donde se observan una organización con tamaño 10, otra grado 4, 4 más grado 3 y las 9 restantes grado 2 .

Al observar de qué tipo son las organizaciones del entorno presentes en las estructuras centrales, se confirman los resultados observados al abordar las redes y su entorno como totalidad: las organizaciones del entorno más importantes para los núcleos de las redes son organizaciones públicas, en particular del orden distrital. 
Este hecho evidencia que dichas organizaciones tienden a poseer una gran influencia sobre las organizaciones vinculadas a ellas. En este sentido el rol de la institucionalidad pública distrital como dinamizador en la realización de acciones y proyectos que favorezcan la emergencia de gestión compartida entre organizaciones, podría configurarse en un punto fuerte para el desarrollo de las redes sociales en la localidad de Ciudad Bolívar, y por qué no, en la ciudad de Bogotá D.C.

\section{Conclusión}

En primer lugar, según los resultados anteriores, cabe resaltar que en la localidad de Ciudad Bolívar se identificaron redes de organizaciones en torno a la gestión de proyectos y acciones compartidas, en el periodo 2005-2006. Esta observación inicial posee un valor fundamental en tanto que el ARS realizado se hizo en función de lograr un diagnóstico actual acerca de la dinámica relacional entre las organizaciones que trabajan en la localidad; y que dicho diagnóstico pueda ser un aporte estratégico hacia la formulación e implementación de una política pública distrital para la construcción de una red de organizaciones entorno a la reconciliación en Bogotá D.C. Por lo tanto, más allá de las características específicas de la red hay que reconocer que, en alguna medida, la gestión compartida es una estrategia de acción aplicada por algunas organizaciones en la localidad, y que este hecho ofrece a los formuladores de política un camino de entrada posible para estimular el desarrollo de dichas redes.

No obstante, algunas de las características comunes a las redes de proyectos y acciones compartidas observadas indican retos o situaciones que la política pública difícilmente podría evitar. En primer lugar, que dichas redes tiendan a un tamaño pequeño en comparación al número de organizaciones potenciales en la localidad; y segundo, que entre aquellas que participan de forma efectiva en la red se observa una tendencia a establecer pocos vínculos y, por lo tanto, las redes en su totalidad son poco densas.

En tanto al tamaño de las redes la señal parece evidente: aunque algunas organizaciones en la localidad utilizan una estrategia de gestión que contempla la posibilidad de asociarse con otras para la realización de acciones y proyectos compartidos, este comportamiento tiende a ser excepcional. De hecho entre las organizaciones identificadas como potenciales para el ARS, e incluso entre las participantes efectivas en la recolección de información, la mayor parte no había realizado ningún tipo de gestión compartida en el lapso 2005-2006. En específico, 
el $74 \%$ de las organizaciones entrevistadas declaró no tener proyectos compartidos, mientras que $54 \%$ declaró lo mismo en relación a las acciones. Si las causas de esta situación pueden atribuirse a una cultura organizacional, en la cual los miembros de las organizaciones prefieren trabajar de forma aislada que asociada con otras organizaciones; o si se puede atribuir a una respuesta de las organizaciones al actual entorno local, distrital o, incluso, nacional, el cual no estimulara el trabajo compartido; o por una combinación de estos y otros motivos posibles, deja planteada una problemática interesante para desarrollar en proyectos de investigación futuros asociados al tema.

En relación a la densidad de las redes, es fundamental resaltar que su potencial se encuentra muy poco desarrollado. Según la baja densidad tanto de las redes proyectos como de acciones compartidas, se pueden caracterizar como relativamente frágiles, de acuerdo a que su capacidad para conectar a las organizaciones involucradas es utilizada de forma mínima. Redes poco densas poseen un riesgo latente de desaparecer dado el caso en que los vínculos existentes entre las organizaciones se agoten y no se creen otros nuevos. En este sentido, cada vínculo de acciones o proyectos compartidos entre las organizaciones participante es un aporte muy relevante a la densificación de la totalidad de la red. Por lo tanto estimular la creación y el desarrollo de vínculos sería un objetivo relevante en una política pública dirigida al desarrollo de estas redes sociales.

De forma adicional, el bajo grado de densidad de estas redes se puede interpretar como una señal de que entre las organizaciones participantes la gestión compartida es una forma válida de actuar, más no necesariamente la opción más estratégica o relevante. En este sentido, en tanto que la participación promedio de las organizaciones en las redes presentan resultados según los cuales estas tienden a contar con apenas un vínculo. Así las cosas, la participación en más de un vínculo, por parte de alguna organización social, refleja un comportamiento que ya se puede ver como excepcional en comparación al promedio general.

No obstante, cabe resaltar algunas particularidades de estas redes para hacer evidentes algunos puntos fuertes en su interior. Primero, se encuentra que la gestión de acciones compartidas es una opción más popular que la de proyectos compartidos. Segundo, que la participación de instituciones públicas del orden distrital y de autoridades locales puede considerarse como un factor clave de articulación e integración de distintos tipos de organizaciones tanto en las redes de acciones como de proyectos compartidos. Y por último, que según el objeto de trabajo de las organizaciones participantes en las redes, se identifica que el 
fomento de actividades culturales y artísticas configura un subgrupo de organizaciones que tiende a ser más dinámico que el resto en términos del establecimiento de vínculos entorno a la gestión compartida.

Con respecto al primer punto, que las organizaciones de las redes realicen en mayor cantidad acciones que proyectos compartidos, indica una tendencia a preferir conexiones menos formales y más coyunturales. A pesar de que esto podría interpretarse como una debilidad, es relativo en tanto que no necesariamente los proyectos compartidos deben considerarse como el motor principal de integración entre las organizaciones. Además, es probable que a través del fomento y apoyo, que pueda brindar una política pública, a la realización de una mayor cantidad de acciones compartidas se derive progresivamente en la generación de vínculos más fuertes y estables, que se traduzcan en futuros proyectos conjuntos. Esto es relevante en tanto se considere que la confianza y las conexiones entre las organizaciones son algo que se desarrolla más de manera progresiva que instantánea, y sobre lo cual la política puede tener un grado de ingerencia relevante.

De acuerdo a lo anterior, el segundo punto acerca de la participación destacada de las instituciones distritales y las autoridades locales en las redes observadas cobra importancia. Es clave, hacer evidente que en buena medida las organizaciones públicas y las autoridades locales participantes son actores articuladores de las redes. Esta situación se podría reconocer y fortalecer como una estrategia política deliberada, la cual implicaría que con un esfuerzo de coordinación interinstitucional se estimule tanto la participación de una mayor cantidad de organizaciones a las redes, como la generación de un mayor número de vínculos entre sus participantes. Aunque no se indagó de forma específica al respecto, es probable que la importancia de las instituciones públicas y las autoridades locales en la red se relacione con un rol de fuentes de recursos para otras organizaciones, en la realización de algunas de sus acciones y proyectos. Así aunque también sería pertinente ahondar en el sentido, impacto y tipos de participación de las organizaciones públicas en las redes sociales de este tipo, es básico reconocer que son actores con influencia efectiva sobre su estructura y evolución.

Por último, acerca del subgrupo de organizaciones asociadas al fomento del arte y la cultura, es apropiado tener en cuenta que es este sector, en especial, donde se identifica la mayor dinámica relacional en términos de la realización de proyectos y acciones compartidas. Por tanto una política pública que busque apoyar el fomento a una red de organizaciones podría apoyarse y apoyar aquellas organizaciones que 
evidencian una preferencia especial por la realización de acciones y proyectos compartidos con otras. Además en tanto que el objetivo de la red se relaciona con un concepto tan abarcador como lo es la reconciliación, las organizaciones asociadas al sector del arte y la cultura podrían considerarse un tipo de actor líder y dinamizador, entre los distintos tipo posibles de organizaciones participantes, para el desarrollo de estas redes.

\section{Bibliografía}

Acotto, L. (2003). Las organizaciones de la sociedad civil. Un camino para la construcción de ciudadanía, Espacio Editorial, Argentina.

Borgatti, S. Everett, M. \& Freeman, L. (2002). Ucinet for Windows: Software for Social Network Analysis, Harvard: Analytic Technologies.

Departamento Administrativo Nacional de Estadística - DANE- (2005). Proyecciones 2005, Censo Nacional de Población, www.dane.gov.co

DANE-Departamento Administrativo de Planeación Distrital -DAPD- (2003), Encuesta de calidad de vida 2003, DAPD, Bogotá.

Hanneman, R. (2001). Introduccion a los Métodos Del Análisis de Redes Sociales. Capitulo Sexto: Centralidad y Poder. Departamento de Sociología de la Universidad de California. http://wizard.ucr.edu/ rhannema/networks/text/textindex.html

Knoke, D. \& Kaufman, N. (1994). "Organizational Power", en Knoke, D., Political Networks. The structural perspective. Structural analysis in social sciences No. 4, Cambridge University Press, USA.

Palacio, D. Parias, A. Garavito, L. Lulle, T. Duque, M. Gómez, Sh. \& Hurtado, R. (2005). "El enfoque de redes socio ambientales: una propuesta para la identificación y caracterización de actores vinculados a la gestión de la conservación in situ" en Arango, N. (ed), Bases para el diseño de sistemas regionales de áreas protegidas, Instituto de Investigación de Recursos Biológicos Alexander von Humbolt, Bogotá.

Palacio, D. Hurtado, R \& Garavito, L. (2003). “Redes Socio-ambientales en tensión: El caso de la gestión ambiental de los humedales de Bogotá", en Revista hispana para el análisis de redes sociales. Vol.X, enero 2002, (http://members.es.tripod.de/revistaredes/html-vol1/vol1_6.htm)

Secretaria de Gobierno Distrital, -SGD- (2005). Diagnóstico Localidad 19, Alcaldía Mayor de Bogotá, Bogotá.

Villar, R. (2003). “De la participación a la incidencia de las organizaciones de la sociedad civil en políticas públicas", en Bombal, I. \& Villar, R. (Comp), Organizaciones de la sociedad civil e incidencia en políticas públicas, Libros del Zorsal, Argentina.

Wasserman, S. y Faust, K. (1994). Social network analysis. Methods and application. Structural analysis in the social sciences No. 8, Cambridge University press, USA. 